\title{
Unterschätzte Krankheit Nykturie: Neuer Lösungsansatz für Patienten
}

— Altersabhängig leiden etwa 30-45\% der Männer über 50 Jahre an therapiebedürftigen Miktionsstörungen. Dies ergab eine für Deutschland repräsentative Studie, die vom Arbeitskreis Benigne Prostatahyperplasie der Akademie der Deutschen Urologen initiiert wurde, erklärte Dr. Richard Berges, Köln.

Nykturie ist nicht nur das häufigste Einzelsymptom bei ansonsten gesunden Männern, es ist auch eines der häufigsten Begleitsymptome bei anderen Erkrankungen, die zu Miktionsstörungen führen. Interessant ist, dass die Ursachen für Nykturie bei Männern und Frauen offensichtlich unterschiedlich bewertet werden. Während Nykturie bei Frauen meistens einer überaktiven Blase (Overactive Bladder, OAB) zugeordnet wird, teilt man sie bei Männern dem benignen Prostatasyndrom zu so Berges. Dabei wird häufig vergessen, dass Nykturie auch Symptom Blasen- oder Prostata-ferner Erkrankungen wie Diabetes mellitus, Diabetes insipidus, Polydipsie oder kardialen Problemen ist. Zudem kommt Nykturie auch als Symptom einer eigenen Erkrankung, der nächtlichen Polyurie vor. Die Differentialdiagnose der Nykturie ist somit unabdingbar für eine erfolgreiche Therapie. Daneben muss ein weiterer Aspekt der Nykturie berücksichtigt werden: Die Tagesmüdigkeit als Folge des gestörten ersten Tiefschlafs. Ein therapeutischer Ansatz muss auch darauf abzielen, die erste Tiefschlafphase in der Nacht zu verlängern, also das Intervall bis zum ersten Aufstehen zur Miktion. Sowohl traditionelle BPS-Medikamente wie Alpha1-Rezeptorenblocker als auch die inzwischen häufiger eingesetzte Kombination mit Anticholinergika haben bei Männern mit $O A B$ ist nicht in jedem Fall den gewünschten Erfolg. Eine Alternative ist Desmopressin, ein synthetisches Analogon des antidiuretischen Hormons. Es wirkt vornehmlich an den renalen $V_{2}$-Rezeptoren und führt daher in geringerem Maße als Vasopressin zu va- sopressorischen und uterotonischen Nebenwirkungen. Wegen des günstigenWirkprofils und seinem schnellem Wirkeintritt eignet sich Desmopressin gut zur medikamentösen Therapie der nächtlichen Polyurie, so Berges. Da nächtliche Polyurie häufig auch in Begleitung mit anderen Erkrankungen auftritt, liegt es nahe, dass die Kombination von Desmopression mit einer Therapie der OAB oder des BPS eingesetztwerden kann. Dies muss in der Zukunft in Studienprotokollen untersucht werden. Bei Männern mit BPS oder OAB gilt bereits jetzt, dass Kombinationstherapie besser ist als Monotherapie, so Berges. Mit Desmopressin steht ein weiterer Therapiebaustein zur Verfügung, um gerade das führende Einzelsymptom Nykturie besser in den Griff zu bekommen.

Pressegespräch „Ferring in der Urologie: neue Maßstäbs, alterspezifische Therapiekonzepte im Rahmen der DGU, Dresden, 18. September 2009; Veranstalter: Ferring

\section{Schmerztherapie: Stabile orale Basisanalgesie verbessert auch den Schlaf}

— „Schmerzen erhöhen das Risiko für Schlafstörungen um das Drei- bis Vierfache“, erklärte Dr. Johannes Horlemann, Kevelaer. Vor allem Einschlafstörung, Fragmentation oder Früherwachen verkürzen die Schlafdauer. Die Folge: Tagesfrische und kognitive Leistungsfähigkeit sind reduziert, die Schmerzwahrnehmung verstärkt.

Einen Ausweg kann laut Horlemann die Einstellung auf Stufe-III-Opioide bieten, insbesondere auf oral wirksames, verzögert freigesetztes Hydromorphon (Jurnista ${ }^{\circledR}$ ). Das retardierte Opioid erzeugt bei ein Mal täglicher Gabe über 24 Stunden gleichmäßige Plasmaspiegel. Die stabile Basisanalgesie verhindert Durchbruch- beziehungsweise End-of-dose-Schmerzen.

Erreicht wird der Effekt über die so genannte OROS $^{\circledR}$-Technologie: Dabei nimmt der Patient ein osmotisch aktives System wie eine Tablette ein; nach Aufnahme in den Magen-Darm-Trakt dringt Wasser in das System, bringt dort einen Quellkörper zur Ausdehnung, so dass Hydromorphon kontinuierlich über eine kleine, lasergebohrte
Öffnung in der Hülle freigesetzt wird. Das System ist auf 24 Stunden justiert, wird danach inaktiv und ausgeschieden. Gleichmäßige Plasmaspiegel zeigen sich innerhalb von zwei Tagen. Die Pharmakokinetik ist in allen Dosierungen $(8,16,32,64 \mathrm{mg})$ dosisproportional.

Bei den 113 von Ärzten befragten Schmerzpatienten besserte sich die Schlafqualität nach Umstellung auf das retardierte Hydromorphon deutlich: Nur noch jeder Zehnte von zuvor jedem Zweiten gab Schlafzeiten von weniger als fünf Stunden an. Fragmentierten Schlaf berichteten noch 46\% (zuvor 91\%). $73 \%$ fühlten sich nach dem Erwachen nun erholt und frisch, zuvor waren dies lediglich 13\%. Die stabile Analgesie reduziert darüber hinaus den Bedarf an Akutmedikation und Schlafmitteln.

koc

Pressegespräch „Therapieziel: Lückenlose Schmerzkontrolle - 24 Stunden, Tag und Nacht" im Rahmen des Deutscher Schmerzkongresses, Berlin, 8. Oktober 2009, Veranstalter: Janssen-Cilag

\section{Spezielle Unterhose fördert Wundheilung}

- Eine Zirkumzision wird nicht nur aus kulturell-religiosen Gründen durchgeführt. Oft ist sie aus medizinischen Gründen notwendig. So ist beispielsweise bei männlichen Diabetikern die Anzahl von PhimoseFällen sehr hoch. Nicht selten wird ein chirurgischer Eingriff notwen-

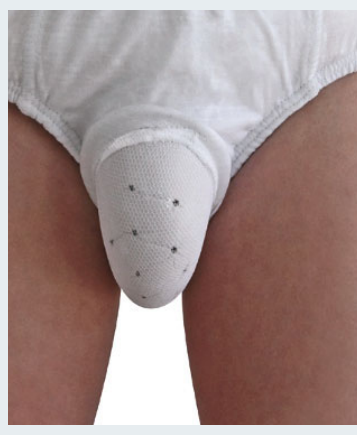
dig. AYDON ${ }^{\circledR}$-Beschneidungsunterhosen ${ }^{\circledR}$ sollen anschließend die Wundheilungsprozess fördern, ein Produkt, das sich in der Türkei bewährt hat. Die Unterhose aus 100\% Baumwolle ist mit einem Protektorkörpchen aus Silikon versehen, das atmungsaktiv ist und gleichzeitig die Wunde schützt. Informationen unter www.aydon.org.

Nach Informationen Aydon 